\title{
Masses of doubly heavy-quark baryons in an extended chromomagnetic model
}

\author{
Xin-Zhen Weng, ${ }^{1,2, *}$ Xiao-Lin Chen, ${ }^{1, \dagger}$ and Wei-Zhen Deng ${ }^{1,2, \sharp}$ \\ ${ }^{1}$ School of Physics, Peking University, Beijing 100871, China \\ ${ }^{2}$ State Key Laboratory of Nuclear Physics and Technology, Peking University, Beijing 100871, China
}

(Received 29 January 2018; published 12 March 2018)

\begin{abstract}
We extend the chromomagnetic model by further considering the effect of color interaction. The effective mass parameters between quark pairs $\left(m_{q q}\right.$ or $\left.m_{q \bar{q}}\right)$ are introduced to account for both the effective quark masses and the color interaction between the two quarks. Using the experimental masses of hadrons, the quark pair parameters are determined between the light quark pairs and the light-heavy quark pairs. Then the parameters of heavy quark pairs $(c c, c b, b b)$ are estimated based on simple quark model assumption. We calculate all masses of doubly and triply heavy-quark baryons. The newly discovered doubly charmed baryon $\Xi_{c c}$ fits into the model with an error of $12 \mathrm{MeV}$.
\end{abstract}

DOI: 10.1103/PhysRevD.97.054008

\section{INTRODUCTION}

In 2002, the SELEX Collaboration [1] reported the first observation of a doubly charmed baryon $\Xi_{c c}^{+}$in the decay mode $\Xi_{c c}^{+} \rightarrow \Lambda_{c}^{+} K^{-} \pi^{+}$. Its mass was determined to be $3519 \pm 1 \mathrm{MeV}$. Further works identified its isospin partner $\Xi_{c c}^{++}$(3460) [2] and an excited state $\Xi_{c c}^{++}$(3780) [3]. Later the SELEX Collaboration confirmed the $\Xi_{c c}^{+}$state in the $\Xi_{c c}^{+} \rightarrow p D^{+} K^{-}[4,5]$ and $\Xi_{c c}^{+} \rightarrow \Xi_{c}^{+} \pi^{+} \pi^{-}[6]$ decay modes. However, none of these states were confirmed by other experimental collaborations [7-10] in the subsequent searches. Recently, the LHCb Collaboration [11] reported the observation of $\Xi_{c c}^{++}$in the $\Lambda_{c}^{+} K^{-} \pi^{+} \pi^{+}$decay mode. But its mass was determined to be $3621.40 \pm 0.72$ (stat.) \pm 0.27 (syst.) $\pm 0.14\left(\Lambda_{c}^{+}\right) \mathrm{MeV}$.

In contrast to the rarity of the experimental observation of the doubly heavy baryons, there is a vast literature of theoretical studies concerning the doubly and even triply heavy baryons with different approaches, including quark models [12-22], QCD sum rules [23-27], lattice QCD [28-43], the bag model [44], heavy-quark effective theory [45], heavy-quark spin symmetry [46], effective field theory with potential nonrelativistic QCD [47,48], the Feynman-Hellmann theorem [49], variational method

\footnotetext{
*xzhweng@pku.edu.cn †chenxl@pku.edu.cn

¥dwz@pku.edu.cn
}

Published by the American Physical Society under the terms of the Creative Commons Attribution 4.0 International license. Further distribution of this work must maintain attribution to the author(s) and the published article's title, journal citation, and DOI. Funded by SCOAP ${ }^{3}$.
[50], the Skyrmion model [51], and the Regge phenomenology $[52,53]$.

The quark model is one of the most used approaches to study the mass spectra of hadrons [12,54-67]. In the nonrelativistic limit, the QCD interaction can be reduced to the potential interaction between quarks. Usually the potential interaction in a quark model consists of the spin-independent color interaction including the linear confinement and Coulomb-type terms, plus higher order terms such as the spin-spin chromomagnetic interaction, tensor interaction, and spin-orbit interactions.

When focusing on lowest $S$-wave states of hadrons, one may adopt the chromomagnetic model [12,68-77]. The chromomagnetic model assumes a mass formula by simply adding a term of chromomagnetic hyperfine interaction to the effective quark masses. This simplified model gives a good account of the hyperfine splittings in hadron mass spectra and produces many useful Gell-Mann-Okubo (GMO) mass relations. From the point of view of the quark model, the effective quark masses also include the chromoelectric effects from the color interaction. However it is difficult to account for the two-body chromoelectric effects in all relevant mesons and baryons by the effective quark masses, which are one-body type. In Ref. [78], Høgaasen et al. generalized the chromomagnetic model by including a chromoelectric term $H_{\mathrm{CE}}=-\sum_{i, j} A_{i j} \tilde{\lambda}_{i} \cdot \tilde{\lambda}_{j}$. Similarly, Karliner et al. introduced the color-singlet binding energies $B(c \bar{c})=-242.7 \mathrm{MeV}$ and $B(b \bar{b})=$ $-532.2 \mathrm{MeV}$ [79].

In this paper, we use the extended chromomagnetic model with the chromoelectric term to study the mass spectra of all the lowest $S$-wave doubly and triply heavyquark baryons systematically. In Sec. II we introduce the extended chromomagnetic model and construct the model 
wave functions of mesons and baryons. In Sec. III A we determine the model parameters. The numerical results are presented and discussed in Sec. III B. We conclude in Sec. IV.

\section{THE EXTENDED CHROMOMAGNETIC MODEL}

\section{A. The Hamiltonian}

In the quark model, the quark effective Hamiltonian reads $[63,64]$

$$
H=H_{0}+\sum_{i<j} V_{i j}
$$

where

$$
H_{0}=\sum_{i} \sqrt{\mathbf{p}_{i}^{2}+m_{i}^{2}}
$$

is the relativistic mass term and $V_{i j}$ is the quark interaction potential between $i$ th and $j$ th quarks. In a nonrelativistic reduction,

$$
H_{0} \rightarrow \sum_{i}\left(m_{i}+\frac{\mathbf{p}_{i}^{2}}{2 m_{i}}\right)
$$

and

$$
V_{i j} \rightarrow V_{i j}^{\text {conf }}+V_{i j}^{\text {hyp }}+V_{i j}^{\text {so }}
$$

where

$$
V_{i j}^{\text {conf }}=-\left[\frac{3}{4} c+\frac{3}{4} b r-\frac{\alpha_{s}(r)}{r}\right] \boldsymbol{F}_{i} \cdot \boldsymbol{F}_{j}
$$

includes the color linear confinement and the Coulombtype interaction, $V_{i j}^{\mathrm{hyp}}$ is the color hyperfine interaction, and $V_{i j}^{\mathrm{so}}$ is the spin-orbit interaction. For the $S$-wave hadron, $V_{i j}^{\mathrm{so}}$ has no contribution, and $V_{i j}^{\text {hyp }}$ can be simply replaced by the chromomagnetic interaction

$$
V_{i j}^{\mathrm{cm}}=-\frac{8 \pi}{3} \frac{\alpha_{s}(r)}{m_{i} m_{j}} \delta^{3}(\boldsymbol{r}) \boldsymbol{S}_{i} \cdot \boldsymbol{S}_{j} \boldsymbol{F}_{i} \cdot \boldsymbol{F}_{j},
$$

where $\boldsymbol{S}_{i}$ and $\boldsymbol{F}_{i}$ are the $i$ th quark's spin operator and color operator, respectively,

$$
\boldsymbol{F}_{i}= \begin{cases}+\frac{\lambda_{i}}{2} & \text { for quarks } \\ -\frac{\lambda_{i}^{*}}{2} & \text { for antiquarks. }\end{cases}
$$

In the case of the lowest $S$-wave hadron, one may further simplify the chromomagnetic interaction by ignoring its spatial dependency. Then the chromomagnetic model Hamiltonian reads

$$
H=\sum_{i} m_{i}+\sum_{i<j} V_{i j}^{\mathrm{cm}}
$$

where the effective mass $m_{i}$ of $i$ th constituent quark (or antiquark) should include the constituent quark mass and the kinetic energy and chromoelectric effects from $V_{i j}^{\text {conf }}$. The chromomagnetic interaction reads

$$
V_{i j}^{\mathrm{cm}}=-v_{i j} \boldsymbol{S}_{i} \cdot \boldsymbol{S}_{j} \boldsymbol{F}_{i} \cdot \boldsymbol{F}_{j}
$$

The coefficient $v_{i j}$ depends on the quark masses and the spatial wave function of the hadron

$$
v_{i j}=\frac{8 \pi}{3 m_{i} m_{j}}\left\langle\alpha_{s}(r) \delta^{3}(\boldsymbol{r})\right\rangle .
$$

However it is difficult to adsorb all the two-body chromoelectric effects into the one-body effective quark masses if we want to study all lowest $S$-wave mesons and baryons together [78,79]. In Ref. [78], Høgaasen et al. generalized the chromomagnetic model by including a chromoelectric term

$$
H_{\mathrm{CE}}=-\sum_{i, j} A_{i j} \tilde{\lambda}_{i} \cdot \tilde{\lambda}_{j}
$$

where $\tilde{\lambda}_{i}=2 \boldsymbol{F}_{i}$. We use this extended chromomagnetic model to study all lowest $S$-wave mesons and baryons systematically.

Since

$$
\begin{aligned}
\sum_{i<j} & \left(m_{i}+m_{j}\right) \boldsymbol{F}_{i} \cdot \boldsymbol{F}_{j} \\
= & \frac{1}{2} \sum_{i, j}\left(m_{i}+m_{j}\right) \boldsymbol{F}_{i} \cdot \boldsymbol{F}_{j}-\sum_{i} m_{i} \boldsymbol{F}_{i}^{2} \\
& =\left(\sum_{i} m_{i} \boldsymbol{F}_{i}\right) \cdot\left(\sum_{i} \boldsymbol{F}_{i}\right)-\frac{4}{3} \sum_{i} m_{i},
\end{aligned}
$$

and the color operator $\sum_{i} \boldsymbol{F}_{i}$ nullifies any colorless physical state, we can introduce a new mass parameter of quark pair

$$
m_{i j}=\left(m_{i}+m_{j}\right)+\frac{16}{3} A_{i j} .
$$

Then the model Hamiltonian reads

$$
H_{\mathrm{CM}}=-\frac{3}{4} \sum_{i<j} m_{i j} V_{i j}^{\mathrm{C}}-\sum_{i<j} v_{i j} V_{i j}^{\mathrm{CM}},
$$

where we have briefly introduced two operators to represent the color and chromomagnetic (CM) interactions between quarks,

$$
\begin{gathered}
V_{i j}^{\mathrm{C}}=\boldsymbol{F}_{i} \cdot \boldsymbol{F}_{j}, \\
V_{i j}^{\mathrm{CM}}=\boldsymbol{S}_{i} \cdot \boldsymbol{S}_{j} \boldsymbol{F}_{i}^{a} \cdot \boldsymbol{F}_{j}^{a} .
\end{gathered}
$$

For the mesons the Hamiltonian is simplified to

$$
H_{\mathrm{CM}}=m_{q \bar{q}}-v_{q \bar{q}} V_{q \bar{q}}^{\mathrm{CM}},
$$


and for the baryons

$$
H_{\mathrm{CM}}=\frac{1}{2} \sum_{i<j} m_{i j}-\sum_{i<j} v_{i j} V_{i j}^{\mathrm{CM}} .
$$

Since the quark model parameters of the baryon system usually are different from that of the meson system, we assume that the pair parameters $m_{q q}$ and $v_{q q}$ are different from their partners $m_{q \bar{q}}$ and $v_{q \bar{q}}$, respectively. Their relations are studies in the next section, based on the numerical analysis and the quark model consideration.

\section{B. Mesons}

A meson is a color-singlet hadron composed of a quark and an antiquark. Its total spin is either 1 or 0 . The corresponding spin wave functions are denoted by

$$
\chi_{1 m}=\left\{|\uparrow \uparrow\rangle, \frac{1}{\sqrt{2}}(|\uparrow \downarrow\rangle+|\downarrow \uparrow\rangle),|\downarrow \downarrow\rangle\right\},
$$

$$
\chi_{00}=\frac{1}{\sqrt{2}}(|\uparrow \downarrow\rangle-|\downarrow \uparrow\rangle),
$$

where $m$ is the third component of the total spin.

The masses of the pseudoscalar and vector mesons are given by

$$
\begin{gathered}
M_{J=0}=m_{q \bar{q}}-v_{q \bar{q}}, \\
M_{J=1}=m_{q \bar{q}}+\frac{1}{3} v_{q \bar{q}} .
\end{gathered}
$$

\section{Baryons}

Baryons are composed of three quarks. Since we only consider the lowest $S$-wave baryons and the color wave function is antisymmetric, we only have to construct the symmetric spin $\otimes$ flavor wave functions.

The total spin of the baryon can be either $3 / 2$ or $1 / 2$. The spin wave functions are classified according to the permutation symmetry,

$$
\begin{gathered}
\chi_{\frac{3}{2} m}^{\mathrm{S}}=\left\{|\uparrow \uparrow \uparrow\rangle, \frac{1}{\sqrt{3}}(|\uparrow \uparrow \downarrow\rangle+|\uparrow \downarrow \uparrow\rangle+|\downarrow \uparrow \uparrow\rangle), \frac{1}{\sqrt{3}}(|\uparrow \downarrow \downarrow\rangle+|\downarrow \uparrow \downarrow\rangle+|\downarrow \downarrow \uparrow\rangle),|\downarrow \downarrow \downarrow\rangle\right\}, \\
\chi_{\frac{1}{2} m}^{\mathrm{MS}}=\left\{-\frac{1}{\sqrt{6}}(|\uparrow \downarrow \uparrow\rangle+|\downarrow \uparrow \uparrow\rangle-2|\uparrow \uparrow \downarrow\rangle), \frac{1}{\sqrt{6}}(|\uparrow \downarrow \downarrow\rangle+|\downarrow \uparrow \downarrow\rangle-2|\downarrow \downarrow \uparrow\rangle)\right\}, \\
\chi_{\frac{1}{2} m}^{\mathrm{MA}}=\left\{\frac{1}{\sqrt{2}}(|\uparrow \downarrow \uparrow\rangle-|\downarrow \uparrow \uparrow\rangle), \frac{1}{\sqrt{2}}(|\uparrow \downarrow \downarrow\rangle-|\downarrow \uparrow \downarrow\rangle)\right\},
\end{gathered}
$$

where the superscript MS (MA) suggests the symmetric (antisymmetric) property of the wave functions under the exchange of the first two quarks.

Next, we combine the flavor wave functions $\left|q_{1} q_{2} q_{3}\right\rangle$ with the spin wave functions. We get the following spin $\otimes$ flavor base wave functions:

$$
\begin{gathered}
J=3 / 2: \phi_{\frac{3}{2} m}^{\left\{q_{1} q_{2} q_{3}\right\}}=|\{q q q\}\rangle \otimes \chi_{\frac{3}{2} m}^{\mathrm{S}}, \\
J=1 / 2: \phi_{\frac{1}{2} m}^{\left\{q_{1} q_{2}\right\} q_{3}}=\left|\left\{q_{1} q_{2}\right\} q_{3}\right\rangle \otimes \chi_{\frac{1}{2} m}^{\mathrm{MS}}+\text { permutations }, \\
\phi_{\frac{1}{2} m}^{\left[q_{1} q_{2}\right] q_{3}}=\left|\left[q_{1} q_{2}\right] q_{3}\right\rangle \otimes \chi_{\frac{1}{2} m}^{\mathrm{MA}}+\text { permutations, }
\end{gathered}
$$

where we use the brace $\{\cdots\}$ to symmetrize the quark flavors and the bracket $[\cdots]$ to antisymmetrize the flavors.

The mass of the spin- $\frac{3}{2}$ baryon is given by

$$
M_{J=\frac{3}{2}}=\frac{1}{2}\left(m_{q_{1} q_{2}}+m_{q_{1} q_{3}}+m_{q_{2} q_{3}}\right)+\frac{1}{6}\left(v_{q_{1} q_{2}}+v_{q_{1} q_{3}}+v_{q_{2} q_{3}}\right) .
$$

To obtain the masses of the spin- $\frac{1}{2}$ baryons consisting of three different quark flavors, we need to diagonalize the following $2 \times 2$ matrix in the above basis [Eqs. (27) and (28)],

$$
H_{J=\frac{1}{2}}=\frac{1}{2}\left(m_{q_{1} q_{2}}+m_{q_{1} q_{3}}+m_{q_{2} q_{3}}\right)+\left(\begin{array}{cc}
\frac{1}{6}\left(v_{q_{1} q_{2}}-2 v_{q_{1} q_{3}}-2 v_{q_{2} q_{3}}\right) & -\frac{1}{2 \sqrt{3}}\left(v_{q_{1} q_{3}}-v_{q_{2} q_{3}}\right) \\
-\frac{1}{2 \sqrt{3}}\left(v_{q_{1} q_{3}}-v_{q_{2} q_{3}}\right) & -\frac{1}{2} v_{q_{1} q_{2}}
\end{array}\right),
$$

which gives us two mixed states, which we denote by $\phi_{\frac{1}{2} m}^{q_{1} q_{2} q_{3} \pm}$, with masses

$$
M_{J=\frac{1}{2}}^{ \pm}=\frac{1}{2}\left(m_{q_{1} q_{2}}+m_{q_{1} q_{3}}+m_{q_{2} q_{3}}\right)-\frac{1}{6}\left(v_{q_{1} q_{2}}+v_{q_{1} q_{3}}+v_{q_{2} q_{3}}\right) \pm \Delta_{J=\frac{1}{2}}
$$


respectively, where

$$
\Delta_{J=\frac{1}{2}}=\frac{1}{3} \sqrt{v_{q_{1} q_{2}}^{2}+v_{q_{1} q_{3}}^{2}+v_{q_{2} q_{3}}^{2}-v_{q_{1} q_{2}} v_{q_{1} q_{3}}-v_{q_{1} q_{2}} v_{q_{2} q_{3}}-v_{q_{1} q_{3}} v_{q_{2} q_{3}}} .
$$

TABLE I. Baryon assignments.

\begin{tabular}{|c|c|c|c|c|}
\hline Flavor & Spin- $\frac{1}{2}$ baryon & Assignment & Spin- $\frac{3}{2}$ baryon & Assignment \\
\hline$n n n$ & $N$ & $\phi_{\frac{1}{2} m}^{\{n n\} n}$ & $\Delta$ & $\phi_{\frac{3}{2} m}^{\{n n n\}}$ \\
\hline \multirow[t]{2}{*}{$n n s$} & $\Sigma$ & $\phi_{\frac{1}{2} m}^{\{n n\} s}$ & $\Sigma^{*}$ & $\phi_{\frac{3}{2} m}^{\{n n s\}}$ \\
\hline & $\Lambda$ & $\phi_{\frac{1}{2} m}^{[n n] s}$ & & \\
\hline$n s s$ & $\Xi$ & $\phi_{\frac{1}{2} m}^{\{s s\} n}$ & $\Xi^{*}$ & $\phi_{\frac{1}{2} m}^{\{n s s\}}$ \\
\hline$s s s$ & & & $\Omega$ & $\phi_{\frac{3}{2} m}^{\{s s s\}}$ \\
\hline \multirow[t]{2}{*}{$n n c$} & $\Sigma_{c}$ & $\phi_{\frac{1}{2} m}^{\{n n\} c}$ & $\Sigma_{c}^{*}$ & $\phi_{\frac{3}{2} m}^{\{n n c\}}$ \\
\hline & $\Lambda_{c}$ & $\phi_{\frac{1}{2} m}^{[n n] c}$ & & \\
\hline \multirow[t]{2}{*}{$n s c$} & $\Xi_{c}$ & $\phi_{\frac{1}{2} m}^{n s c-}$ & $\Xi_{c}^{*}$ & $\phi_{\frac{3}{2} m}^{\{n s c\}}$ \\
\hline & $\Xi_{c}^{\prime}$ & $\phi_{\frac{1}{2} m}^{n s c+}$ & & \\
\hline$s s c$ & $\Omega_{c}$ & $\phi_{\frac{1}{2} m}^{\{s s\} c}$ & $\Omega_{c}^{*}$ & $\phi_{\frac{3}{2} m}^{\{s s c\}}$ \\
\hline \multirow[t]{2}{*}{$n n b$} & $\Sigma_{b}$ & $\phi_{\frac{1}{2} m}^{\{n n\} b}$ & $\Sigma_{b}^{*}$ & $\phi_{\frac{3}{2} m}^{\{n n b\}}$ \\
\hline & $\Lambda_{b}$ & $\phi_{\frac{1}{2} m}^{[n n] b}$ & & \\
\hline \multirow[t]{2}{*}{$n s b$} & $\Xi_{b}$ & $\phi_{\frac{1}{2} m}^{n s b-}$ & $\Xi_{b}^{*}$ & $\phi_{\frac{3}{2} m}^{\{n s b\}}$ \\
\hline & $\Xi_{b}^{\prime}$ & $\phi_{\frac{1}{2} m}^{n s b+}$ & & \\
\hline$s s b$ & $\Omega_{b}$ & $\phi_{\frac{1}{2} m}^{\{s s\} b}$ & $\Omega_{b}^{*}$ & $\phi_{\frac{3}{2} m}^{\{s s b\}}$ \\
\hline$n c c$ & $\Xi_{c c}$ & $\phi_{\frac{1}{2} m}^{\{c c\} n}$ & $\Xi_{c c}^{*}$ & $\phi_{\frac{3}{2} m}^{\{n c c\}}$ \\
\hline$s c c$ & $\Omega_{c c}$ & $\phi_{\frac{1}{2} m}^{\{c c\} s}$ & $\Omega_{c c}^{*}$ & $\phi_{\frac{3}{2} m}^{\{s c c\}}$ \\
\hline$c c c$ & & & $\Omega_{c c c}$ & $\phi_{\frac{3}{2} m}^{\{c c c\}}$ \\
\hline$n b b$ & $\Xi_{b b}$ & $\phi_{\frac{1}{2} m}^{\{b b\} n}$ & $\Xi_{b b}^{*}$ & $\phi_{\frac{3}{2} m}^{\{n b b\}}$ \\
\hline$s b b$ & $\Omega_{b b}$ & $\phi_{\frac{1}{2} m}^{\{b b\} s}$ & $\Omega_{b b}^{*}$ & $\phi_{\frac{3}{2} m}^{\{s b b\}}$ \\
\hline$b b b$ & & & $\Omega_{b b b}$ & $\phi_{\frac{3}{2} m}^{\{b b b\}}$ \\
\hline \multirow[t]{2}{*}{$n c b$} & $\Xi_{c b}$ & $\phi_{\frac{1}{2} m}^{n c b-}$ & $\Xi_{c b}^{*}$ & $\phi_{\frac{3}{2} m}^{\{n c b\}}$ \\
\hline & $\Xi_{c b}{ }^{\prime}$ & $\phi_{\frac{1}{2} m}^{n c b+}$ & & \\
\hline \multirow[t]{2}{*}{$s c b$} & $\Omega_{c b}$ & $\phi_{\frac{1}{2} m}^{s c b-}$ & $\Omega_{c b}^{*}$ & $\phi_{\frac{3}{2} m}^{\{s c b\}}$ \\
\hline & $\Omega_{c b}^{\prime}$ & $\phi_{\frac{1}{2} m}^{s c b+}$ & & \\
\hline$c c b$ & $\Omega_{c c b}$ & $\phi_{\frac{1}{2} m}^{\{c c\} b}$ & $\Omega_{c c b}^{*}$ & $\phi_{\frac{3}{2} m}^{\{c c b\}}$ \\
\hline$c b b$ & $\Omega_{c b b}$ & $\phi_{\frac{1}{2} m}^{\{b b\} c}$ & $\Omega_{c b b}^{*}$ & $\phi_{\frac{3}{2} m}^{\{c b b\}}$ \\
\hline
\end{tabular}

Note that if the flavors of any two quarks in the baryon are identical, we can assign $q_{1}=q_{2}$ and the second combination [Eq. (28)] does not exist. Then we get only one spin- $\frac{1}{2}$ baryon state $\phi_{\frac{1}{2} m}^{\left\{q_{1} q_{1}\right\} q_{3}}$ with mass

$$
E_{J=\frac{1}{2}}=\frac{1}{2}\left(m_{q_{1} q_{1}}+2 m_{q_{1} q_{3}}\right)+\frac{1}{6}\left(v_{q_{1} q_{1}}-4 v_{q_{1} q_{3}}\right) .
$$

We collect the wave function assignments of all lowest $S$-wave baryons in Table I.

\section{NUMERICAL RESULTS}

\section{A. Parameters}

First we consider the mesons. We can extract the two parameters $m_{q_{1} \bar{q}_{2}}$ and $v_{q_{1} \bar{q}_{2}}$ from the experimental masses of corresponding $q_{1} \bar{q}_{2}$ pseudoscalar and vector mesons. For the $n \bar{n}$ mesons consisting of $u, d$ flavors, we only use the isovector $\pi$ and $\rho$ mesons to extract $m_{n \bar{n}}$ and $v_{n \bar{n}}$.

We do not consider $\eta$ and $\eta^{\prime}$ mesons to avoid the complexity of flavor octet-singlet mixing and the chiral anomaly. Instead, we use the following PCAC (partially conserved axial current) result [80-82],

$$
M_{s \bar{s}\left({ }^{1} S_{0}\right)}=\sqrt{2 M_{K}^{2}-M_{\pi}^{2}}=687.220 \mathrm{MeV}
$$

and the experimental mass of the $\phi$ meson to extract the parameters $m_{s \bar{s}}$ and $v_{s \bar{s}}$. The equation can also be derived in the chiral perturbation theory [83].

Another difficulty is that only one of the two $c \bar{b}$ states, that is, the $B_{c}$ meson, was observed in experiment. This state was first reported by CDF and OPAL collaborations in 1998 $[84,85]$, whose current mass in PDG is $6275.1 \mathrm{MeV}$ [86]. Godfrey et al. had predicted its mass to be $6.27 \mathrm{GeV}$ using the quark model in 1985 [63]; a more detailed study in 2004 gives $M_{B_{c}}=6271 \mathrm{MeV}$ [65], which is very closed to the experimental value. They also predicted $M_{B_{c}^{*}}=6338 \mathrm{MeV}$. Other quark model calculation coincides with their result. For instance, Ikhdair et al. [87] predict $M_{B_{c}^{*}}=6340 \mathrm{MeV}$ and Ebert et al. [88] predict $M_{B_{c}^{*}}=6332 \mathrm{MeV}$. In our work, we use the prediction $M_{B_{c}^{*}}=6338 \mathrm{MeV}$ of Godfrey et al. to determine the parameters of the $c \bar{b}$ pair. All the $q \bar{q}$ pair parameters are presented in Table II.

Now we turn to the baryon sector. We can only use the experimental masses of light-quark baryons and singly heavy-quark baryons to extract the model parameters. 
TABLE II. Parameters of $q \bar{q}$ pairs (in unit of $\mathrm{MeV}$ ).

\begin{tabular}{lccccccccc}
\hline \hline$m_{n \bar{n}}$ & $m_{n \bar{s}}$ & $m_{s \bar{s}}$ & $m_{n \bar{c}}$ & $m_{s \bar{c}}$ & $m_{c \bar{c}}$ & $m_{n \bar{b}}$ & $m_{s \bar{b}}$ & $m_{c \bar{b}}$ & $m_{b \bar{b}}$ \\
615.95 & 794.22 & 936.40 & 1973.22 & 2076.14 & 3068.53 & 5313.35 & 5403.25 & 6322.27 & 9444.97 \\
& & & & & & & & & \\
$v_{n \bar{n}}$ & $v_{n \bar{s}}$ & $v_{s \bar{s}}$ & $v_{n \bar{c}}$ & $v_{s \bar{c}}$ & $v_{c \bar{c}}$ & $v_{n \bar{b}}$ & $v_{s \bar{b}}$ & $v_{c \bar{b}}$ & $v_{b \bar{b}}$ \\
477.92 & 298.57 & 249.18 & 106.01 & 107.87 & 85.12 & 33.89 & 36.43 & 47.18 & 45.98 \\
\hline \hline
\end{tabular}

TABLE III. Parameters of light-light and light-heavy quark pairs with statistical errors (in units of MeV).

\begin{tabular}{lccccc}
\hline \hline$m_{n n}$ & $m_{n s}$ & $m_{n c}$ & $m_{s c}$ & $m_{n b}$ & $m_{s b}$ \\
$724.85 \pm 3.37$ & $906.65 \pm 3.43$ & $2079.96 \pm 4.47$ & $2183.68 \pm 5.33$ & $5412.25 \pm 4.81$ & $5494.80 \pm 10.05$ \\
$v_{n n}$ & $v_{n s}$ & $v_{n c}$ & $v_{s c}$ & $v_{n b}$ & $v_{s b}$ \\
$305.34 \pm 6.54$ & $212.75 \pm 6.06$ & $62.81 \pm 9.68$ & $70.63 \pm 9.92$ & $19.92 \pm 10.19$ & $8.47 \pm 16.66$ \\
$m_{s s}+v_{s s} / 3$ & & & & & \\
$1114.45 \pm 4.55$ & & & & & \\
\hline \hline
\end{tabular}

Besides, the $\Omega_{b}^{*}$ has not yet been observed in experiment. We perform an unweighted nonlinear least-squares fit of 23 known baryon masses to extract 13 model parameters, using the GSL library [89]. Note that, with two identical quarks, the pair parameters $m_{q q}$ and $v_{q q}$ only appear in the combination $m_{q q}+v_{q q} / 3$ in the mass formulas (33) and (29). So we can only determine the value $m_{s s}+v_{s s} / 3$ from the experimental data.

The baryon parameters obtained are presented in Table III. The fitting standard deviation is $7.66 \mathrm{MeV}$. Because the $\Omega_{b}^{*}$ has not yet been observed in experiment, the parameters $m_{s b}$ and $v_{s b}$ have large statistical errors. The comparison of the fitted mass values with experimental data is listed in Table IV. Most fitting deviations of the baryon masses are within $10 \mathrm{MeV}$. The only exception is the $\Sigma$, whose deviation is $15.0 \mathrm{MeV}$.

In our model, all chromoelectric effects of color interaction are included in the pair mass parameter $m_{q q}$ (or $m_{q \bar{q}}$ ). If the chromoelectric effects can be absorbed into the quark mass $m_{q}$ like in the original chromomagnetic model, we have the relation

$$
m_{q_{1} \bar{q}_{1}}+m_{q_{2} \bar{q}_{2}}-2 m_{q_{1} \bar{q}_{2}} \approx 0
$$

This is not true from our fitting. Typically

TABLE IV. Comparison for light and singly heavy-quark baryon masses (with statistical errors) with experimental data [86] (in units of $\mathrm{MeV}$ ).

\begin{tabular}{|c|c|c|c|c|c|}
\hline & $n n n$ & $n n s$ & $n n s$ & $n s s$ & sss \\
\hline$J^{P}=1 / 2^{+}$ & $N(938.9)$ & $\Sigma(1193.2)$ & $\Lambda(1115.7)$ & $\Xi(1318.3)$ & $\cdots$ \\
\hline theo. & $934.6 \pm 6.0$ & $1178.1 \pm 5.7$ & $1116.4 \pm 5.0$ & $1322.1 \pm 5.8$ & \\
\hline$J^{P}=3 / 2^{+}$ & $\Delta(1232)$ & $\Sigma^{*}(1384.6)$ & $\ldots$ & $\Xi^{*}(1533.4)$ & $\Omega(1672.5)$ \\
\hline \multirow[t]{2}{*}{ theo. } & $1239.9 \pm 6.0$ & $1390.9 \pm 4.5$ & & $1534.8 \pm 4.6$ & $1671.7 \pm 6.8$ \\
\hline & $n n c$ & $n n c$ & $n s c$ & $n s c$ & $s s c$ \\
\hline$J^{P}=1 / 2^{+}$ & $\Sigma_{c}(2453.6)$ & $\Lambda_{c}(2286.5)$ & $\Xi_{c}^{\prime}(2576.8)$ & $\Xi_{c}(2469.4)$ & $\Omega_{c}(2695.2)$ \\
\hline theo. & $2451.4 \pm 8.1$ & $2289.7 \pm 5.8$ & $2576.2 \pm 5.6$ & $2478.7 \pm 5.6$ & $2693.8 \pm 8.8$ \\
\hline$J^{P}=3 / 2^{+}$ & $\Sigma_{c}^{*}(2518.1)$ & $\ldots$ & $\Xi_{c}^{*}(2645.9)$ & $\ldots$ & $\Omega_{c}^{*}(2765.9)$ \\
\hline \multirow[t]{2}{*}{ theo. } & $2514.2 \pm 5.9$ & & $2642.8 \pm 4.6$ & & $2764.5 \pm 6.7$ \\
\hline & $n n b$ & $n n b$ & $n s b$ & $n s b$ & $s s b$ \\
\hline$J^{P}=1 / 2^{+}$ & $\Sigma_{b}(5813.4)$ & $\Lambda_{b}(5619.5)$ & $\Xi_{b}^{\prime}(5935.0)$ & $\Xi_{b}(5793.2)$ & $\Omega_{b}(6046.4)$ \\
\hline theo. & $5812.3 \pm 8.6$ & $5622.0 \pm 6.1$ & $5932.9 \pm 7.8$ & $5800.4 \pm 7.8$ & $6046.4 \pm 15.1$ \\
\hline$J^{P}=3 / 2^{+}$ & $\Sigma_{b}^{*}(5833.6)$ & $\cdots$ & $\Xi_{b}^{*}(5952.1)$ & $\cdots$ & $\Omega_{b}^{*}$ \\
\hline theo. & $5832.2 \pm 6.2$ & & $5947.0 \pm 6.8$ & & $6054.8 \pm 11.7$ \\
\hline
\end{tabular}


TABLE V. Difference of pair mass parameters extracted from baryons and mesons (in units of $\mathrm{MeV}$ ).

\begin{tabular}{lccccc}
\hline \hline$\delta m_{n n}$ & $\delta m_{n s}$ & $\delta m_{n c}$ & $\delta m_{s c}$ & $\delta m_{n b}$ & $\delta m_{s b}$ \\
\hline $108.89 \pm 3.37$ & $112.44 \pm 3.43$ & $106.74 \pm 4.47$ & $107.54 \pm 5.33$ & $98.90 \pm 4.81$ & $91.54 \pm 10.05$ \\
\hline \hline
\end{tabular}

TABLE VI. Quark mass difference $\delta m_{q}$ (in units of $\mathrm{MeV}$ ).

\begin{tabular}{lccc}
\hline \hline$\delta m_{n}$ & $\delta m_{s}$ & $\delta m_{c}$ & $\delta m_{b}$ \\
\hline $54.94 \pm 1.51$ & $56.48 \pm 3.06$ & $51.49 \pm 3.68$ & $42.30 \pm 4.51$ \\
\hline \hline
\end{tabular}

$$
m_{n \bar{n}}+m_{b \bar{b}}-2 m_{n \bar{b}} \approx-600 \mathrm{MeV}
$$

We also note that the quark pair mass $m_{q q}$ is quite different from $m_{q \bar{q}}$ of its quark antiquark partner. We list the difference $\delta m_{q_{1} q_{2}} \equiv m_{q_{1} q_{2}}-m_{q_{1} \bar{q}_{2}}$ in Table V. Indeed, many authors found that the effective quark masses extracted from baryons were larger than that from mesons $[18,75,79,90]$. This mass difference can be also accounted by adjusting the constant $c$ in the quark interaction [Eq. (5)], if it can be treated as a constant $[63,64]$. Here we assume that

$$
A_{q \bar{q}} \approx A_{q q}
$$

in Eq. (13) and the difference of the pair mass parameter becomes

$$
\delta m_{q_{1} q_{2}} \equiv m_{q_{1} q_{2}}-m_{q_{1} \bar{q}_{2}} \approx \delta m_{q_{1}}+\delta m_{q_{2}}
$$

where $\delta m_{q}=m_{q}^{b}-m_{q}^{m}$ is the difference of the effective quark mass extracted from the baryon and meson. Then we perform a least-squares fitting to obtain the mass difference $\delta m_{q}$, which is listed in Table VI. The reduced chi-squared statistic is $\chi_{\nu}^{2}=0.41$.

In Table VII, we compare the chromomagnetic interaction strengths in baryons and mesons using their ratio
$R_{q_{1} q_{2}} \equiv v_{q_{1} q_{2}} / v_{q_{1} \bar{q}_{2}}$. We find that $R_{n n}, R_{n s}, R_{n c}, R_{s c}$ are very close to each others. $R_{s b}$ is relatively small but with large statistical error due to the lack of experimental data of $B_{c}^{*}$. This phenomenon was first observed by Keren-Zur [91]. The ratio was interpreted in the quark model, using the Cornell potential or the Logarithmic potential. The author also gave a simple interpretation by assuming that the contact probability in the chromomagnetic interaction [Eq. (6)] is inversely proportional to the number of quarks in the hadron. Since the quark number is 3 in a baryon and 2 in a meson, this gives a rough estimate of $R_{q_{1} q_{2}} \approx 2 / 3$. To estimate the heavy quark pair parameters $\left\{v_{c c}, v_{c b}, v_{b b}\right\}$, we assume that

$$
R_{Q_{1} Q_{2}}=2 / 3 \pm 0.30,
$$

where we use the largest statistical error in Table VII (except $R_{s b}$ whose statistical error is mainly due to the lack of experimental data) to set the parameter range. We should point out that even the estimate causes large standard errors in $\left\{v_{c c}, v_{c b}, v_{b b}\right\}$; it does not have so many significant effects on the mass of doubly and triply heavy-quark baryons as the absolute values $v_{Q_{1} Q_{2}}$ are much smaller than $v_{q Q}$ between light and heavy quarks.

Using the mass difference Eq. (36) and ratio relation Eq. (37), we can determine the parameters between two heavy quarks, as well as $m_{s s}$ and $v_{s s}$. All the baryon parameters are collected in Table VIII.

\section{B. Mass spectra of doubly and triply heavy baryons}

Substituting the parameters obtained in Sec. III A into the Hamiltonians, we can obtain the masses of doubly and

TABLE VII. Ratio of CM interaction strength $R_{q_{1} q_{2}}=v_{q_{1} q_{2}} / v_{q_{1} \bar{q}_{2}}$.

\begin{tabular}{ccccccc}
\hline \hline$q_{1} q_{2}$ & $n n$ & $n s$ & $n c$ & $s c$ & $n b$ & $s b$ \\
\hline Ratio & $0.64 \pm 0.01$ & $0.71 \pm 0.02$ & $0.59 \pm 0.09$ & $0.65 \pm 0.09$ & $0.59 \pm 0.30$ & $0.23 \pm 0.46$ \\
\hline \hline
\end{tabular}

TABLE VIII. Parameters of $q q$ pairs (in units of $\mathrm{MeV}$ ).

\begin{tabular}{lcccc}
\hline \hline$m_{n n}$ & $m_{n s}$ & $m_{s s}$ & $m_{n c}$ & $m_{s c}$ \\
$724.85 \pm 3.37$ & $906.65 \pm 3.43$ & $1049.36 \pm 4.32$ & $2079.96 \pm 4.47$ & $2183.68 \pm 5.33$ \\
$m_{c c}$ & $m_{n b}$ & $m_{s b}$ & $m_{c b}$ & $m_{b b}$ \\
$3171.51 \pm 5.21$ & $5412.25 \pm 4.81$ & $5494.80 \pm 10.05$ & $6416.07 \pm 5.82$ & $9529.57 \pm 6.37$ \\
$v_{n n}$ & $v_{n s}$ & $v_{s s}$ & $v_{n c}$ & $v_{s c}$ \\
$305.34 \pm 6.54$ & $212.75 \pm 6.06$ & $195.30 \pm 18.84$ & $62.81 \pm 9.68$ & $70.63 \pm 9.92$ \\
$v_{c c}$ & $v_{n b}$ & $v_{s b}$ & $v_{c b}$ & $v_{b b}$ \\
$56.75 \pm 25.54$ & $19.92 \pm 10.19$ & $8.47 \pm 16.66$ & $31.45 \pm 14.15$ & $30.65 \pm 13.79$ \\
\hline \hline
\end{tabular}


TABLE IX. Mass of the doubly and triply heavy baryons (in units of MeV).

\begin{tabular}{|c|c|c|c|c|c|c|}
\hline & $n c c$ & $s c c$ & $c c c$ & $n b b$ & $s b b$ & $b b b$ \\
\hline$J^{P}=1 / 2^{+}$ & $\Xi_{c c}$ & $\Omega_{c c}^{+}$ & $\cdots$ & $\Xi_{b b}$ & $\Omega_{b b}^{-}$ & $\cdots$ \\
\hline Exp. & $3518.7 \pm 1.7^{\mathrm{a}}$ & & & & & \\
\hline Exp. & $3621.40 \pm 0.72^{b}$ & & & & & \\
\hline Theo. & $3633.3 \pm 9.3$ & $3731.8 \pm 9.8$ & & $10168.9 \pm 9.2$ & $10259.0 \pm 15.5$ & \\
\hline$J^{P}=3 / 2^{+}$ & $\Xi_{c c}^{*}$ & $\Omega_{c c}^{*+}$ & $\Omega_{c c c}^{*++}$ & $\Xi_{b b}^{*}$ & $\Omega_{b b}^{*-}$ & $\Omega_{b b b}^{*-}$ \\
\hline \multirow[t]{2}{*}{ Theo. } & $3696.1 \pm 7.4$ & $3802.4 \pm 8.0$ & $4785.6 \pm 15.0$ & $10188.8 \pm 7.1$ & $10267.5 \pm 12.1$ & $14309.7 \pm 11.8$ \\
\hline & \multicolumn{2}{|c|}{$n c b$} & \multicolumn{2}{|c|}{$s c b$} & $c c b$ & $c b b$ \\
\hline$J^{P}=1 / 2^{+}$ & $\Xi_{c b}^{\prime}$ & $\Xi_{c b}$ & $\Omega_{c b}^{\prime 0}$ & $\Omega_{c b}^{0}$ & $\Omega_{c c b}^{+}$ & $\Omega_{c b b}^{0}$ \\
\hline Theo. & $6947.9 \pm 6.9$ & $6922.3 \pm 6.9$ & $7047.0 \pm 9.3$ & $7010.7 \pm 9.3$ & $7990.3 \pm 12.2$ & $11165.0 \pm 11.8$ \\
\hline$J^{P}=3 / 2^{+}$ & $\Xi_{c b}^{*}$ & & $\Omega_{c b}^{\prime * 0}$ & & $\Omega_{c c b}^{*+}$ & $\Omega_{c b b}^{* 0}$ \\
\hline Theo. & $6973.2 \pm 5.5$ & & $7065.7 \pm 7.5$ & & $8021.8 \pm 9.0$ & $11196.4 \pm 8.5$ \\
\hline
\end{tabular}

\section{${ }^{\mathrm{a}} \mathrm{SELEX}[1]$. \\ ${ }^{\mathrm{b}} \mathrm{LHCb}[11]$.}

triply heavy-quark baryons. They are summarized in Table IX.

In our calculation $M_{\Xi_{c c}}=3633.3 \pm 9.3 \mathrm{MeV}$. It is much heavier than the SELEX's value by approximately $100 \mathrm{MeV}$ [1], and very closed to the report of LHCb [11]. The $\Xi_{c c}^{*}$ state lies $62.8 \mathrm{MeV}$ above $\Xi_{c c}$. This splitting is very closed to the one between $\Sigma_{c}$ and $\Sigma_{c}^{*}(64.5 \mathrm{MeV})$, which is consistent with the GMO mass relation [92]

$$
M_{\Xi_{c c}^{*}}-M_{\Xi_{c c}}=M_{\Sigma_{c}^{*}}-M_{\Sigma_{c}}
$$

A similar relation holds if we replace the $u, d$ quarks by the $s$ quark

$$
M_{\Omega_{c c}^{*}}-M_{\Omega_{c c}}=M_{\Omega_{c}^{*}}-M_{\Omega_{c}}
$$

where both sides are approximately $71 \mathrm{MeV}$. Similar to the $\Sigma_{c}^{(*)}$ (or $\Omega_{c}^{(*)}$ ) case, the splitting between $\Xi_{c c}^{*}$ and $\Xi_{c c}$ (or between $\Omega_{c c}^{*}$ and $\Omega_{c c}$ ) is too small to induce a transition through the emission of the $\pi$ meson; however, the transition is still possible through $\gamma$ emission.

The situation for bottomed baryons is similar;

$$
M_{\Xi_{b b}^{*}}-M_{\Xi_{c c}} \approx M_{\Sigma_{b}^{*}}-M_{\Sigma_{b}},
$$

where the left-hand side is $19.9 \mathrm{MeV}$ and the right-hand side is $20.2 \mathrm{MeV}$. This splitting is significantly smaller than that of charmed baryons. The reason is that the hyperfine splitting is reciprocal to the masses of quarks, and of course the $b$ quark is much heavier than the $c$ quark.

There is also one GMO mass relation about the triply heavy-quark baryons, that is,

$$
M_{\Omega_{c b b}^{*}}-M_{\Omega_{c b b}}=M_{\Omega_{c c b}^{*}}-M_{\Omega_{c c b}},
$$

where both sides are approximately $31 \mathrm{MeV}$.

For spin-1/2 doubly heavy-quark baryons composed of three different quarks, namely, the $q c b$ baryon states $(q=u, d, s)$, one should consider the mixture between two basis states (27) and (28). Numerically, the mixing matrix in Eq. (30) is given by (in $\mathrm{MeV}$ )

$$
\left(\begin{array}{cc}
-6.7 & 3.3 \\
3.3 & -31.4
\end{array}\right) \text { and }\left(\begin{array}{cc}
-1.5 & 6.6 \\
6.6 & -35.3
\end{array}\right)
$$

for $n c b$ and $s c b$ flavor configurations respectively. The eigenvalues of $n c b$ states are $\{-31.8,-6.2\}$, with eigenvectors $\{-0.13,0.99\}$ and $\{0.99,0.13\}$, and the eigenvalues of $s c b$ states are $\{-36.6,-0.3\}$ with eigenvectors $\{-0.19,0.98\}$ and $\{0.98,0.19\}$. In both cases, the mixing is very small and the mixing mass effects are within $2 \mathrm{MeV}$.

If one ignores the mixing, then the $\Xi_{c b}$ and $\Xi_{c b}^{\prime}$ can be treated as states in the flavor $S U(2)_{n c}$ singlet and triplet representations and the $\Omega_{c b}$ and $\Omega_{c b}^{\prime}$ as states in the $S U(2)_{s c}$ singlet and triplet representations, respectively [21]. The following GMO mass relations hold approximately:

$$
\begin{array}{r}
2 M_{\Xi_{c b}^{*}}+M_{\Xi_{c b}^{\prime}}-3 M_{\Xi_{c b}} \approx 2\left(M_{\Sigma_{c}^{*}}-M_{\Sigma_{c}}\right), \\
2 M_{\Omega_{c b}^{*}}+M_{\Omega_{c b}^{\prime}}-3 M_{\Omega_{c b}} \approx 2\left(M_{\Omega_{c}^{*}}-M_{\Omega_{c}}\right), \\
2\left(M_{\Xi_{c b}^{*}}-M_{\Xi_{c b}^{\prime}}\right)-\left(M_{\Omega_{c b b}^{*}}-M_{\Omega_{c b b}}\right) \approx M_{\Sigma_{b}^{*}}-M_{\Sigma_{b}}, \\
2\left(M_{\Omega_{c b}^{*}}-M_{\Omega_{c b}^{\prime}}\right)-\left(M_{\Omega_{c b b}^{*}}-M_{\Omega_{c b b}}\right) \approx M_{\Omega_{b}^{*}}-M_{\Omega_{b}} .
\end{array}
$$

We find that the errors of all those relations are within $5 \mathrm{MeV}$. 


\section{CONCLUSIONS}

In this work, we generalized the chromomagnetic model by considering the effect of color interaction. According to color algebra, the quark effective mass and the color interaction between quarks are combined into a new quark pair mass parameter. The quark pair parameters between two light quarks and that between light-heavy quarks are determined using the experimental masses of lowest $S$-wave hadrons. The pair parameters between two heavy quarks are estimated from the corresponding pair parameters between the quark and antiquark in mesons, using the mass difference and a ratio relation about the chromomagnetic interaction. We have calculated the mass spectra of the lowest $S$-wave doubly and triply heavy baryons. We obtained $M_{\Xi_{c c}}=3633.3 \pm 9.3 \mathrm{MeV}$, which is close to the report of LHCb. We hope that future experiments in LHCb, BES-III et al. confirm the existence of these states.

\section{ACKNOWLEDGMENTS}

The authors thank S. L. Zhu for helpful comments and discussions. X.Z. W. is grateful to J. F. Jiang, H. S. Li, L. Meng, G. J. Wang, S. J. Wu, and B. Zhou for helpful discussions. This project is supported by the National Natural Science Foundation of China under Grants No. 11621131001 and No. 11575008.
[1] M. Mattson et al. (SELEX Collaboration), Phys. Rev. Lett. 89, 112001 (2002).

[2] J. S. Russ (SELEX Collaboration), in Proceedings, 1st International Workshop on Frontier Science: Charm, Beauty, and CP, Frascati, Italy, October 6-11, 2002 (2002), pp. 652-654 [arXiv:hep-ex/0209075].

[3] M. A. Moinester et al. (SELEX Collaboration), in Proceedings, Advanced Studies Institute, INTAS Monitoring Conference, PRAHA-SPIN 2002, Prague, Czech Republic, July 14-28, 2002; Czech. J. Phys. 53, B201 (2003).

[4] A. Ocherashvili et al. (SELEX Collaboration), Phys. Lett. B 628, 18 (2005).

[5] J. Engelfried (SELEX Collaboration), in Proceedings, 22nd International Conference, INPC 2004, Goeteborg, Sweden, June 27-July 2, 2004; Nucl. Phys. A752, 121 (2005).

[6] J. Engelfried (SELEX Collaboration), in Proceedings, 8th International Workshop, HQL06, Munich, Germany, October 16-20, 2006 eConf C0610161, 003 (2006).

[7] S. P. Ratti, in Proceedings, 5th International Conference on Hyperons, Charm, and Beauty Hadrons (BEACH 2002), Vancouver, Canada, June 25-29, 2002; Nucl. Phys. B, Proc. Suppl. 115, 33 (2003).

[8] B. Aubert et al. (BABAR Collaboration), Phys. Rev. D 74, 011103 (2006).

[9] Y. Kato et al. (Belle Collaboration), Phys. Rev. D 89, 052003 (2014).

[10] R. Aaij et al. (LHCb Collaboration), J. High Energy Phys. 12 (2013) 090.

[11] R. Aaij et al. (LHCb Collaboration), Phys. Rev. Lett. 119, 112001 (2017).

[12] A. De Rújula, H. Georgi, and S. L. Glashow, Phys. Rev. D 12, 147 (1975).

[13] J. D. Bjorken, in Proceedings, International Conference on Hadron Spectroscopy: College Park, Maryland, April 20-22, 1985; AIP Conf. Proc. 132, 390 (1985).

[14] B. Silvestre-Brac, Few Body Syst. 20, 1 (1996).

[15] S. S. Gershtein, V. V. Kiselev, A. K. Likhoded, and A. I. Onishchenko, Phys. Rev. D 62, 054021 (2000).
[16] V. V. Kiselev, A. K. Likhoded, O. N. Pakhomova, and V. A. Saleev, Phys. Rev. D 66, 034030 (2002).

[17] D. Ebert, R. N. Faustov, V. O. Galkin, and A. P. Martynenko, Phys. Rev. D 66, 014008 (2002).

[18] M. Karliner and J. L. Rosner, Phys. Rev. D 90, 094007 (2014).

[19] S. Migura, D. Merten, B. Metsch, and H.-R. Petry, Eur. Phys. J. A 28, 41 (2006).

[20] A. P. Martynenko, Phys. Lett. B 663, 317 (2008).

[21] W. Roberts and M. Pervin, Int. J. Mod. Phys. A 23, 2817 (2008).

[22] T. Branz, A. Faessler, T. Gutsche, M. A. Ivanov, J. G. Körner, V. E. Lyubovitskij, and B. Oexl, Phys. Rev. D 81, 114036 (2010).

[23] J.-R. Zhang and M.-Q. Huang, Phys. Lett. B 674, 28 (2009).

[24] Z.-G. Wang, Eur. Phys. J. A 45, 267 (2010).

[25] L. Tang, X.-H. Yuan, C.-F. Qiao, and X.-Q. Li, Commun. Theor. Phys. 57, 435 (2012).

[26] T. M. Aliev, K. Azizi, and M. Savci, J. Phys. G 40, 065003 (2013).

[27] H.-X. Chen, Q. Mao, W. Chen, X. Liu, and S.-L. Zhu, Phys. Rev. D 96, 031501 (2017).

[28] A. Ali Khan, T. Bhattacharya, S. Collins, C. T. H. Davies, R. Gupta, C. Morningstar, J. Shigemitsu, and J. H. Sloan, Phys. Rev. D 62, 054505 (2000).

[29] R. M. Woloshyn, Phys. Lett. B 476, 309 (2000).

[30] R. Lewis, N. Mathur, and R. M. Woloshyn, Phys. Rev. D 64, 094509 (2001).

[31] J. M. Flynn, F. Mescia, and A. S. B. Tariq (UKQCD Collaboration), J. High Energy Phys. 07 (2003) 066.

[32] T.-W. Chiu and T.-H. Hsieh, in Proceedings, 10th International Conference, Baryons'04, Palaiseau, France, October 25-29, 2004; Nucl. Phys. A755, 471 (2005).

[33] T. Mehen and B.C. Tiburzi, Phys. Rev. D 74, 054505 (2006).

[34] L. Liu, H.-W. Lin, K. Orginos, and A. Walker-Loud, Phys. Rev. D 81, 094505 (2010).

[35] S. Meinel, Phys. Rev. D 82, 114514 (2010). 
[36] C. Alexandrou, J. Carbonell, D. Christaras, V. Drach, M. Gravina, and M. Papinutto, Phys. Rev. D 86, 114501 (2012).

[37] R. A. Briceño, H.-W. Lin, and D. R. Bolton, Phys. Rev. D 86, 094504 (2012).

[38] S. Durr, G. Koutsou, and T. Lippert, Phys. Rev. D 86, 114514 (2012).

[39] Y. Namekawa et al. (PACS-CS Collaboration), Phys. Rev. D 87, 094512 (2013).

[40] M. Padmanath, R. G. Edwards, N. Mathur, and M. Peardon, Phys. Rev. D 91, 094502 (2015).

[41] Z. S. Brown, W. Detmold, S. Meinel, and K. Orginos, Phys. Rev. D 90, 094507 (2014).

[42] J. Vijande, A. Valcarce, and H. Garcilazo, Phys. Rev. D 90, 094004 (2014).

[43] P. Pérez-Rubio, S. Collins, and G. S. Bali, Phys. Rev. D 92 , 034504 (2015).

[44] P. Hasenfratz, R. R. Horgan, J. Kuti, and J. M. Richard, Phys. Lett. 94B, 401 (1980).

[45] J. G. Körner, M. Krämer, and D. Pirjol, Prog. Part. Nucl. Phys. 33, 787 (1994).

[46] J. M. Flynn, E. Hernández, and J. Nieves, Phys. Rev. D 85, 014012 (2012).

[47] N. Brambilla, A. Vairo, and T. Rosch, Phys. Rev. D 72, 034021 (2005).

[48] F. J. Llanes-Estrada, O. I. Pavlova, and R. Williams, Eur. Phys. J. C 72, 2019 (2012).

[49] R. Roncaglia, D. B. Lichtenberg, and E. Predazzi, Phys. Rev. D 52, 1722 (1995).

[50] Y. Jia, J. High Energy Phys. 10 (2006) 073.

[51] M. Rho, D. O. Riska, and N. N. Scoccola, Phys. Lett. B 251, 597 (1990).

[52] L. Burakovsky, J. T. Goldman, and L. P. Horwitz, Phys. Rev. D 56, 7124 (1997).

[53] X.-H. Guo, K.-W. Wei, and X.-H. Wu, Phys. Rev. D 78, 056005 (2008).

[54] Y. Ne'eman, Nucl. Phys. 26, 222 (1961).

[55] M. Gell-Mann, Phys. Rev. 125, 1067 (1962).

[56] M. Gell-Mann, Phys. Lett. 8, 214 (1964).

[57] G. Zweig, in Developments in the Quark Theory of Hadrons Vol. 1. 1964-1978, edited by D. Lichtenberg and S. P. Rosen (1964), pp. 22-101.

[58] E. Eichten, K. Gottfried, T. Kinoshita, K. D. Lane, and T.-M. Yan, Phys. Rev. D 17, 3090 (1978); E. Eichten, K. Gottfried, T. Kinoshita, K. D. Lane, and T.-M. Yan, Phys. Rev. D 21, 313(E) (1980).

[59] C. Quigg and J. L. Rosner, Phys. Lett. 71B, 153 (1977).

[60] A. Martin, Phys. Lett. 100B, 511 (1981).

[61] N. Isgur and G. Karl, Phys. Lett. 72B, 109 (1977).

[62] J. L. Basdevant and S. Boukraa, Z. Phys. C 28, 413 (1985).

[63] S. Godfrey and N. Isgur, Phys. Rev. D 32, 189 (1985).

[64] S. Capstick and N. Isgur, in Proceedings, International Conference on Hadron Spectroscopy: College Park, Maryland, April 20-22, 1985; Phys. Rev. D 34, 2809
(1986); S. Capstick and N. Isgur, AIP Conf. Proc. 132, 267 (1985).

[65] S. Godfrey, Phys. Rev. D 70, 054017 (2004).

[66] S. Godfrey and K. Moats, Phys. Rev. D 93, 034035 (2016).

[67] S. Godfrey, K. Moats, and E. S. Swanson, Phys. Rev. D 94, 054025 (2016).

[68] A. D. Sakharov and Ya. B. Zel'dovich, Yad. Fiz. 4, 395 (1966).

[69] Y. B. Zeldovich and A. D. Sakharov, Sov. J. Nucl. Phys. 4, 283 (1967).

[70] T. A. DeGrand, R. L. Jaffe, K. Johnson, and J. E. Kiskis, Phys. Rev. D 12, 2060 (1975).

[71] R. L. Jaffe, Phys. Rev. D 15, 267 (1977).

[72] R. L. Jaffe, Phys. Rev. D 15, 281 (1977).

[73] B. Silvestre-Brac, Phys. Rev. D 46, 2179 (1992).

[74] Y. Cui, X.-L. Chen, W.-Z. Deng, and S.-L. Zhu, Phys. Rev. D 73, 014018 (2006).

[75] F. Buccella, H. Høgaasen, J.-M. Richard, and P. Sorba, Eur. Phys. J. C 49, 743 (2007).

[76] V. Borka Jovanović, S. R. Ignjatović, D. Borka, and P. Jovanović, Phys. Rev. D 82, 117501 (2010).

[77] J. Wu, Y.-R. Liu, K. Chen, X. Liu, and S.-L. Zhu, Phys. Rev. D 95, 034002 (2017).

[78] H. Høgaasen, E. Kou, J.-M. Richard, and P. Sorba, Phys. Lett. B 732, 97 (2014).

[79] M. Karliner, S. Nussinov, and J. L. Rosner, Phys. Rev. D 95, 034011 (2017).

[80] M. D. Scadron, Phys. Rev. D 26, 239 (1982).

[81] T. Feldmann, in Proceedings, 3rd Euroconference, 6th Conference, Montpellier, France, July 2-8, 1998; Nucl. Phys. B, Proc. Suppl. 74, 151 (1999).

[82] P. Kroll, in Proceedings, 8th International Workshop, MESON 2004, Cracow, Poland, June 4-8, 2004; Int. J. Mod. Phys. A 20, 331 (2005).

[83] S. Scherer and M. R. Schindler, Lect. Notes Phys. 830, 1 (2012).

[84] F. Abe et al. (CDF Collaboration), Phys. Rev. Lett. 81, 2432 (1998).

[85] K. Ackerstaff et al. (OPAL Collaboration), Phys. Lett. B 420, 157 (1998).

[86] C. Patrignani et al. (Particle Data Group), Chin. Phys. C 40, 100001 (2016).

[87] S. M. Ikhdair and R. Sever, Int. J. Mod. Phys. A 19, 1771 (2004).

[88] D. Ebert, R. N. Faustov, and V. O. Galkin, Phys. Rev. D 67, 014027 (2003).

[89] M. Galassi, J. Davies, J. Theiler, B. Gough, G. Jungman, P. Alken, M. Booth, F. Rossi, and R. Ulerich, GNU Scientific Library (2017), https://www.gnu.org/software/gsl/doc/latex/ gsl-ref.pdf.

[90] S. Gasiorowicz and J. L. Rosner, Am. J. Phys. 49, 954 (1981).

[91] B. Keren-Zur, Ann. Phys. (Amsterdam) 323, 631 (2008).

[92] R. J. Johnson, Phys. Rev. D 14, 3220 (1976). 